\title{
Autonomous radar pulse modulation classification using modulation components analysis
}

\author{
Pei Wang ${ }^{*}$, Zhaoyang Qiu, Jun Zhu and Bin Tang
}

\begin{abstract}
An autonomous method for recognizing radar pulse modulations based on modulation components analysis is introduced in this paper. Unlike the conventional automatic modulation classification methods which extract modulation features based on a list of known patterns, this proposed method classifies modulations by the existence of basic modulation components including continuous frequency modulations, discrete frequency codes and discrete phase codes in an autonomous way. A feasible way to realize this method is using the features of abrupt changes in the instantaneous frequency rate curve which derived by the short-term general representation of phase derivative. This method is suitable not only for the basic radar modulations but also for complicated and hybrid modulations. The theoretical result and two experiments demonstrate the effectiveness of the proposed method.
\end{abstract}

Keywords: Autonomous modulation classification, Modulation components analysis, Sliding window, Abrupt changes, Instantaneous frequency rate

\section{Introduction}

Pulse modulation classification plays an essential role in modern intercept receivers for electronic warfare (EW) applications such as threat recognition and analysis, construction of effective jamming responses and radar emitter identification [1]. In the EW context, modulations on radar pulses are divided into two classes, the intentional modulation on pulse (IMOP) and the unintentional modulation on pulse (UMOP). The feature of UMOP is usually applied for the purpose of specific emitter identification while the feature of IMOP is mainly used to collect intelligence of a hostile radar system [2]. Pulse modulation classification is a technique aiming at recognizing the intentional intra-pulse modulations of radar signals and is becoming more and more difficult because of the everincreasing number of emitters and low probability of intercept (LPI) radar waveforms that have appeared in modern electromagnetic environment [3]. The newly emerging radar systems, such as the cognitive radar [4], the waveform-agile radar [5], and the multiple-input multiple-output (MIMO) radar [6,7], are changing their

\footnotetext{
* Correspondence: wangpei1128@foxmail.com

School of Electronic Engineering, University of Electronic Science and Technology of China, Chengdu, China
}

operation modes and bringing waveforms with various kinds of complicated IMOPs. Apart from that, some waveforms with hybrid IMOPs, such as the discrete phase codes on linear frequency modulation (LFM) [8,9], multiple frequency-shift keying (FSK) waveform outperformed by a chirp modulation [10], and the combination of FSK and phase-shift keying (PSK) modulations [11], have also been reported. These complicated and hybrid IMOPs are posing a particular threat to modern EW intercept receivers.

However, nearly all of the existing pulse modulation classification algorithms are realized based on some known emitter databases and are suitable only for a certain group of IMOPs [12]. For example, an atomic decomposition method employing chirplet dictionary was proposed in [13], and it can realize the automatic detection and classification of radar pulses with LFM, PSK, and FSK modulations. An supervised classification system achieving overall correct classification rate of $98 \%$ at signal-to-noise ratio (SNR) of $6 \mathrm{~dB}$ based on multilayer perception networks was proposed in [14] and eight classes of radar signals are classified. Algorithms based on some time-frequency distributions such 
as the ambiguity function (AF) [15], Zhao Altas and Marks (ZAM) representations [16], the Rihacek distribution and the Hough transform [17], were also applied to extract modulation features for five kinds of radar pulses. These existing algorithms work effectively when the radar IMOPs are in the list of their databases, but fail when they are faced with some complicated or unknown modulated pulses.

In this paper, we try to classify radar pulse modulations using an autonomous method. A two-stage classification procedure which contains a "modulation family classification" and an "accurate classification" is proposed. The former part works autonomously based on a modulation components analysis (MCA) method and can extract partial information even when the IMOP of the radar pulse is not in the knowledge database. The latter part is a supplementary part to classify an IMOP in detail and is extensible. The paper is organized as follows. Section 2 gives a universal category of IMOPs and describes signal models using the instantaneous frequency rate (IFR) curves. Section 3 describes the whole classification procedure of the proposed method. Two experiments to demonstrate the effectiveness of the proposed method are designed in Section 4, and Section 5 gives the main conclusions.

\section{Signal model and abrupt changes analysis in IFR curve}

\subsection{Categories of possible radar pulse modulations}

In order to analyze the various kinds of IMOPs conveniently, we categorize them based on three kinds of basic IMOP components including the continuous frequency modulation (CFM), the discrete frequency coded (DFC) modulation and the discrete phase coded (DPC) modulation. Possible IMOPs in CFM class include constant frequency (CF), LFM and various nonlinear frequency modulations (NLFM). The DFC class contains binary frequency codes (BFC), the Costas codes and so on. The DPC class includes IMOPs such as binary phase codes (BPC), quadriphase codes (QPC) and polyphase codes (PPC).

Various modulations can be realized by a single IMOP or combinations of different IMOPs. According to the phase properties between different IMOPs, three modulation families are extended as the CFM family, the DFC family and the DPC family as shown in Fig. 1. But beyond that, a fourth family which contains intra-pulse modulation agile waveform, noise-like waveform and so on may also appear. For the convenience of analysis, here, we consider only the former three families. It is easy to see that not only the common radar pulse modulations that have been used so far, but also the existing and possible complicated or hybrid modulations are under this context.

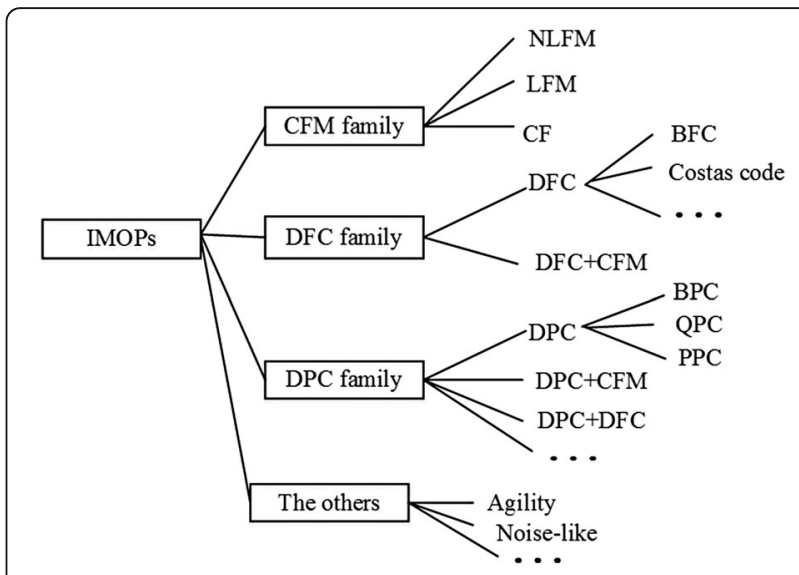

Fig. 1 Possible IMOP families for radar pulses

\subsection{Signal model}

For the intercept receivers, the received radar pulse could be modeled as follows

$$
\begin{aligned}
r(t) & =s(t)+w(t) \\
& =A \exp (j \phi(t))+w(t), \quad 0 \leq t \leq T
\end{aligned}
$$

where $T$ is the pulse duration time, $A$ is the complex amplitude of the signal, $\phi(t)$ is phase of the intercepted radar pulse, and $w(t)$ is the complex additive white Gaussian noise (AWGN) with variance $\sigma^{2}$. The corresponding discrete model is

$$
r(n)=A \exp (j \phi(n))+w(n), \quad n=0, \ldots, N
$$

where the sampling frequency is $f_{s}$.

To identify an unknown, or a complicated modulated radar pulse, we should find out which basic modulation exists and that is why we call the method as "MCA". For a signal in the CFM family, the phase function can be considered as a polynomial phase signal according to the Weierstrass theorem.

$$
\phi_{C F M}(t)=\sum_{k=0}^{P} a_{k} t^{k}, \quad 0 \leq t \leq T
$$

where $\left\{a_{k}\right\}_{k=0, \ldots, P}$ is the polynomial coefficients and $P$ is the highest order of the polynomial coefficients.

For a signal in the DFC family, the basic modulation is frequency coding.

$$
\phi_{\text {BasicDFC }}(t)=\sum_{k=0}^{M-1} 2 \pi f_{k} \operatorname{tg}\left(t-k T_{p}\right), \quad 0 \leq t \leq T
$$

where $M$ is the number of sub-pulses, $f_{k}$ is the carrier frequency of the $k$ th sub-pulse, $T_{p}$ is the width of sub-pulse, and $g(t)$ is a rectangular function and $g(t)=1$ when $0 \leq t<$ $T_{p}$. Considering complicated modulations such as hybrid modulations of CFM and basic DFC components, the phase function of a signal in DFC family can be expressed as 


$$
\phi_{D F C}(t)=\phi_{C F M}(t)+\phi_{\text {BasicDFC }}(t)
$$

For a signal in the DPC family, the basic modulation is phase coding.

$$
\phi_{\text {BasicDPC }}(t)=\sum_{k=0}^{L-1} \theta_{k} g\left(t-k T_{c}\right), \quad 0 \leq t \leq T
$$

where $L$ is the number of phase codes; $\theta_{k}$ is $k$ th phase and $T_{c}$ is the width of phase code. Considering complicated modulations such as hybrid modulations of basic DPC modulations with CFM or DFC modulations, the phase function of a signal in DFC family can be expressed as

$$
\phi_{D P C}(t)=\phi_{C F M}(t)+\phi_{\text {BasicDFC }}(t)+\phi_{\text {BasicDPC }}(t)
$$

The MCA method distinguishes basic modulation components based on the abrupt changes in their phase derivatives. Here the IFR curve is used as example.

\subsection{IFR propertis for basic modulation components}

The direct derivation of IFR is the second derivative of signal phase which can be expressed as:

$$
\operatorname{IFR}(t) \triangleq \frac{1}{2 \pi} \frac{d^{2} \phi(t)}{d t^{2}}
$$

Different modulation families show different properties in their IFR curves. For convenience, three basic modulations which belong to different modulation families are considered here in discrete case.

For the basic modulation in the CFM family, the IFR is shown as

$$
\operatorname{IFR}_{C F M}(n)=\frac{1}{\pi} a_{2}+\zeta(n)
$$

where $\zeta(n)$ is a polynomial phase function. Thus, there are no abrupt changes in the IFR curve. The IFR curve of an LFM pulse is shown in Fig. 2a.
The IFR of a basic DFC modulation is

$$
\operatorname{IFR}(n)=f_{s} \sum_{k=1}^{M}\left(f_{k}-f_{k-1}\right) \delta\left(n-k T_{p}\right)
$$

where $\delta(n)=\left\{\begin{array}{c}1, n=0 \\ 0, \text { otherwise }\end{array}\right.$. Thus, an abrupt change appears if the adjacent sub-pulses have different frequencies and the amplitude of the abrupt change depends on the difference of the two adjacent frequencies. The IFR curve of a Costas coded pulse is shown in Fig. 2b.

The IFR of a basic DPC modulation is

$$
\operatorname{IFR}(n)=\frac{f_{s}^{2}}{2 \pi} \sum_{k=1}^{M}\left(c_{k}-c_{k-1}\right)\left(\delta\left(n-1-k T_{c}\right)-\delta\left(n-k T_{c}\right)\right)
$$

There will be two abrupt changes which have a same absolute value and opposite signs in the IFR curves and the amplitude of the abrupt changes depend on the adjacent phases. The IFR curve of a QPC pulse is shown in Fig. 2c.

A traditional way to approximately realize the phase derivatives is using phase unwrapping and phase difference operations. But, it suffers a rapid degradation of performance even at a high SNR. Figure 3 shows the estimated IFR curves of the same three pulses using the phase-based way at $\mathrm{SNR}=20 \mathrm{~dB}$, and it seems impossible to extract the features of abrupt changes in the IFR curves especially for the DFC signal.

\subsection{Robust IFR estimation and abrupt changes analysis} As the MCA method analyzes the abrupt changes in the IFR curves, methods which are robust to noise and sensitive to abrupt changes need to be found. The IFR estimation was firstly used for chirp signal estimation [18] and the concept of any order phase representation was generalized through the term of general representation of phase derivative (GRPD) [19]. In [20], the IFR
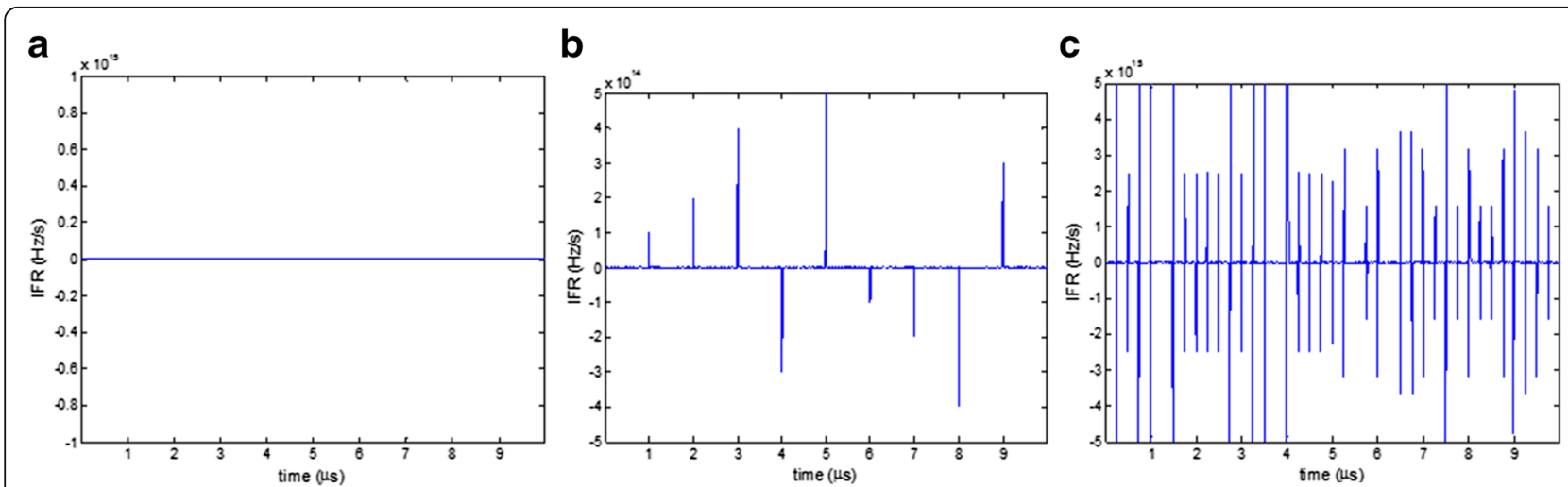

Fig. 2 IFR curves of three basic modulations: (a) LFM; (b) Costas; and (c) QPC 

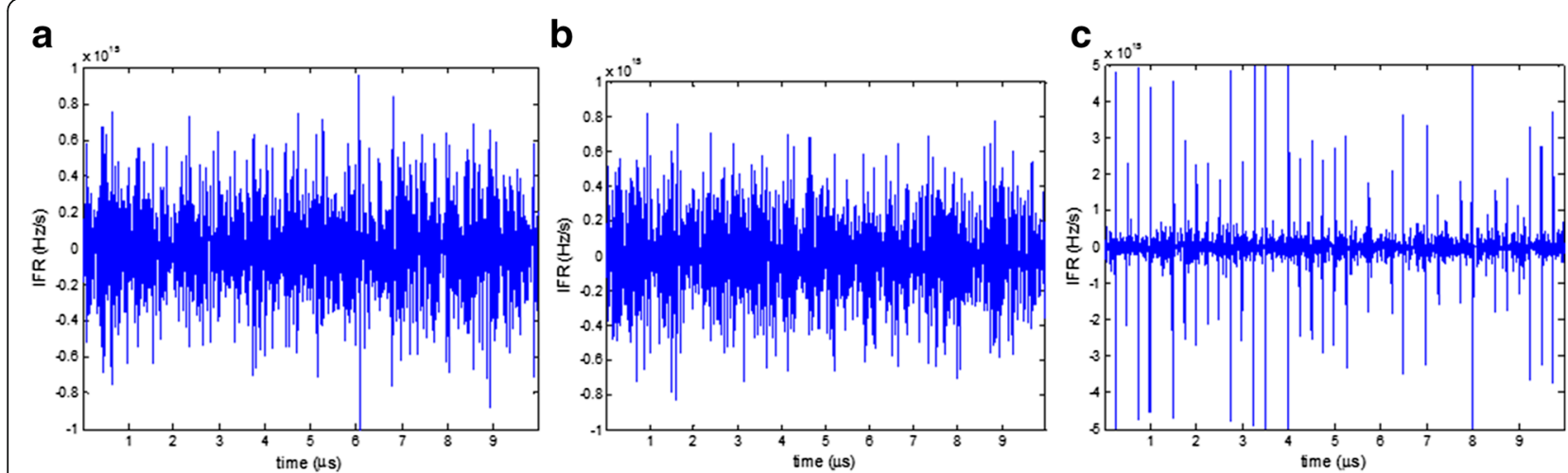

Fig. 3 IFR curves of three basic modulations at SNR = 20 dB: (a) LFM; (b) Costas; and (c) QPC

was derived using the short-term polynomial phase estimation in the EW context and the influence of window length on parameter estimation was analyzed. On the basis of previous studies, we will extract features from the abrupt changes in the IFR curves and then classify the modulation family autonomously in this paper.

The GRPD method is a kind of multi-linear method which is suitable for IFR estimation. It has been proved that the GRPD method using a sliding window has a good performance which approaches the Cramer-Rao bound (CRB) for the IFR estimations [20]. According to the GRPD theory, the ideal representation of an arbitrary instantaneous phase derivative (IPD) of a signal can be written in the form

$$
\operatorname{IPD}_{K}(t, \Omega)=A^{2} \delta\left(\Omega-\phi^{(K)}(t)\right)
$$

where $\Omega$ is the axis which corresponds to the $K$ th derivative of the phase function $\phi(t)$. In order to obtain the distribution concentrated along Kth phase derivative, a generalized complex moment (GCM) is defined as

$$
\operatorname{GCM}_{N}^{K}[s](t, \tau)=\prod_{k=0}^{N-1} s^{\omega_{N, k}^{N-K}}\left(t+\omega_{N, k} \tau\right)
$$

where $\omega_{N, k}=e^{j 2 \pi k / N}$. When $K=1$, the GRPD is the conventional time-frequency distribution.

$$
\operatorname{GCM}_{N}^{1}[s](t, \tau)=\prod_{k=0}^{N-1} s^{\omega_{N, k}^{*}}\left(t+\frac{\omega_{N, k}}{N} \tau\right)
$$

For example, when $N=1$, it leads to the short-time Fourier transform (STFT), and when $N=2$, it leads to a Wigner-Ville-like distribution.

When $K=2$, it is a time-"frequency rate" representation.

$$
\operatorname{GCM}_{N}^{2}[s](t, \tau)=\prod_{k=0}^{N-1} s^{\omega_{N, k}^{N-2}}\left(t+\omega_{N, k} \sqrt{\frac{2}{N} \tau}\right)
$$

To calculate the IFR, here, we consider a simple case for $N=2$. Therefore, the GCMs are

$$
\operatorname{GCM}_{2}^{2}\left[s, t_{i}\right](\tau)=s(t+\sqrt{\tau}) s(t-\sqrt{\tau})
$$

where $\tau \in\left[-T_{w}^{2} / 4, T_{w}^{2} / 4\right]$. For a time instant $t_{i}$, the IFR is estimated using a short-time window by the relations as follows.

$$
\widehat{I F R}\left(t_{i}\right)=\underset{f}{\operatorname{argmax}}\left|\int_{\mathbb{R}} G C M_{2}^{2}\left[s, t_{i}\right](\tau) e^{-j 2 \pi f \tau} d \tau\right|
$$

Supposing that the length of sliding window in calculating the IFR value at a certain time instant is $N_{w}$, it can be approved that [20]

$$
C R B\left\{\operatorname{IFR}\left(t_{i}\right)\right\}=\frac{1}{\pi^{2}} \frac{90 f_{s}^{4}}{N_{w}^{5} S N R} .
$$

Therefore, for a given SNR and a given mean square error (MSE) of estimators, the length of the sliding window should satisfy the following relation.

$$
N_{w} \geq \sqrt[5]{\frac{1}{\pi^{2}} \frac{90 f_{s}^{4}}{\operatorname{MSE}\left\{\operatorname{IFR}\left(t_{i}\right)\right\} S N R}}
$$

Figure 4 shows the estimated IFR curves of the same three pulses in Figs. 2 and 3 at SNR $=3 \mathrm{~dB}$.

It shows that the short-time GRPD method is robust to noise and sensitive to abrupt changes. Therefore, the IFR curves are feasible to realize the autonomous MCA method. 


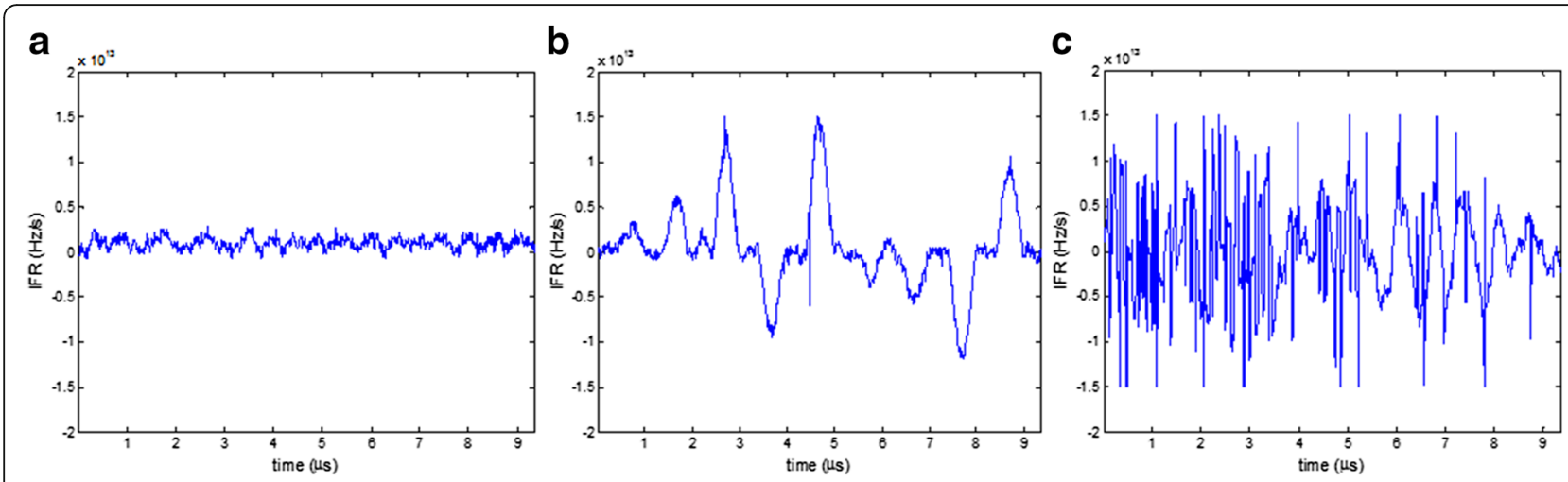

Fig. 4 Estimated IFR curves of three basic modulations at SNR =3 dB: (a) LFM; (b) Costas; and (c) QPC

\section{Autonomous modualtion classification flow based on the MCA method}

The autonomous classification methods focus on the essential differences among the three basic IMOPs. Here, we employ the abrupt changes caused by the DFC and the DPC modulations in the IFR curves. The signal processing flow for radar pulse modulation classification is shown in Fig. 5.

The modulation information is given after three steps. The samples of pulse will be processed to extract some modulation features. Then the MCA method is applied to classify the pulse into a modulation family and this can be also called a "coarse classification". At last, an accurate classification will be done within the modulation family and some possible modulation parameters will be estimated at the same time using existing algorithms.

\subsection{Modulation families classification based on MCA method}

From the analyses above we know that the estimated IFR curve for an IMOP in the CFM family has no abrupt changes. Thus a basic CFM component can be distinguished from the DFC and the DPC families. The estimated IFR curve using a short-time window of a pure DFC components has abrupt changes regularly. Once a change appears, its values will keep positive or negative for a short period. By contrast, the abrupt changes in the estimated IFR curve for a basic DPC component appear mixed and disorderly. According to the differences of abrupt changes, we can recognize the IMOP family of a pulse autonomously. Figure 6 shows the modulation family classification flow based on the MCA method.

The flow chart contains several important modules: SNR estimation and sliding window setting module, IFR estimation module, and abrupt changes analysis module.

\subsubsection{SNR estimation and sliding window setting}

SNR is an important parameter in signal processing. When it comes to a non-cooperative situation, a simple and effective method to estimate the SNR is the 2nd and 4th order moments (M2M4) algorithm [21, 22]. This

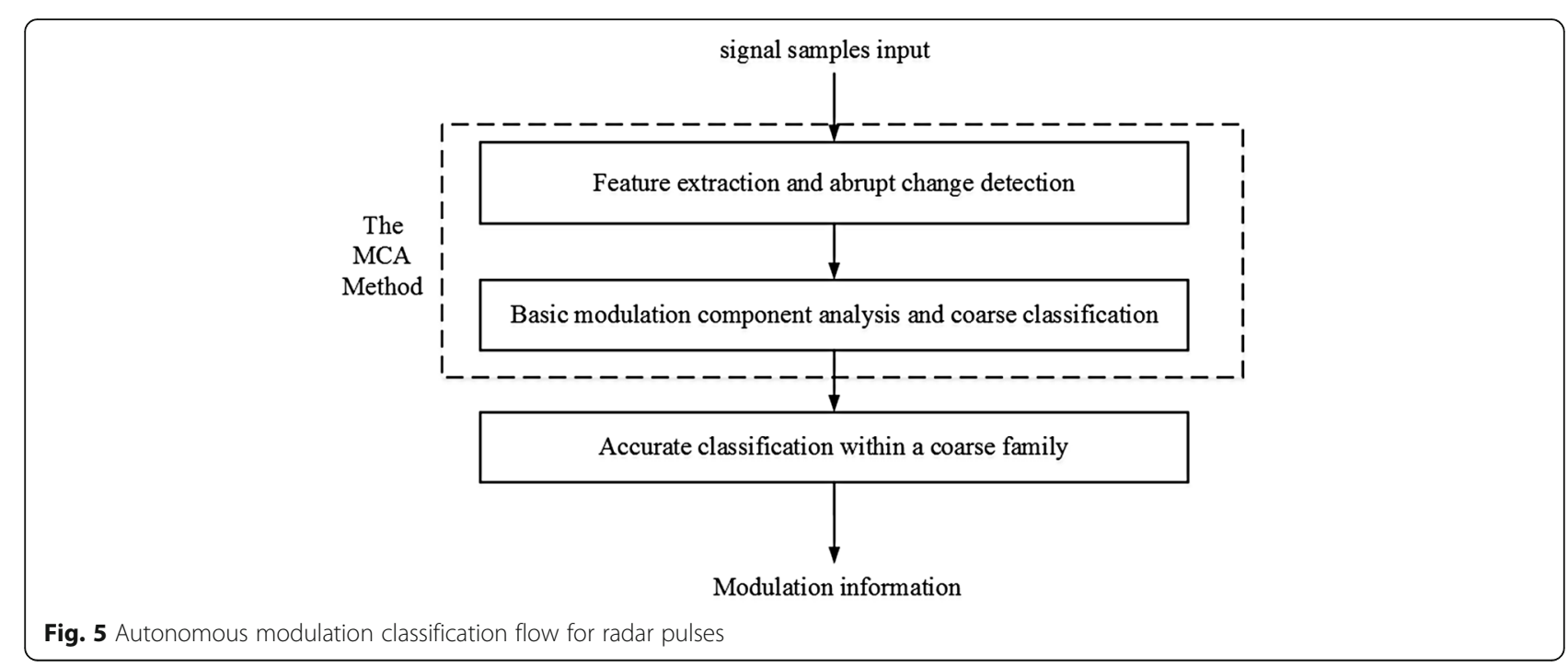




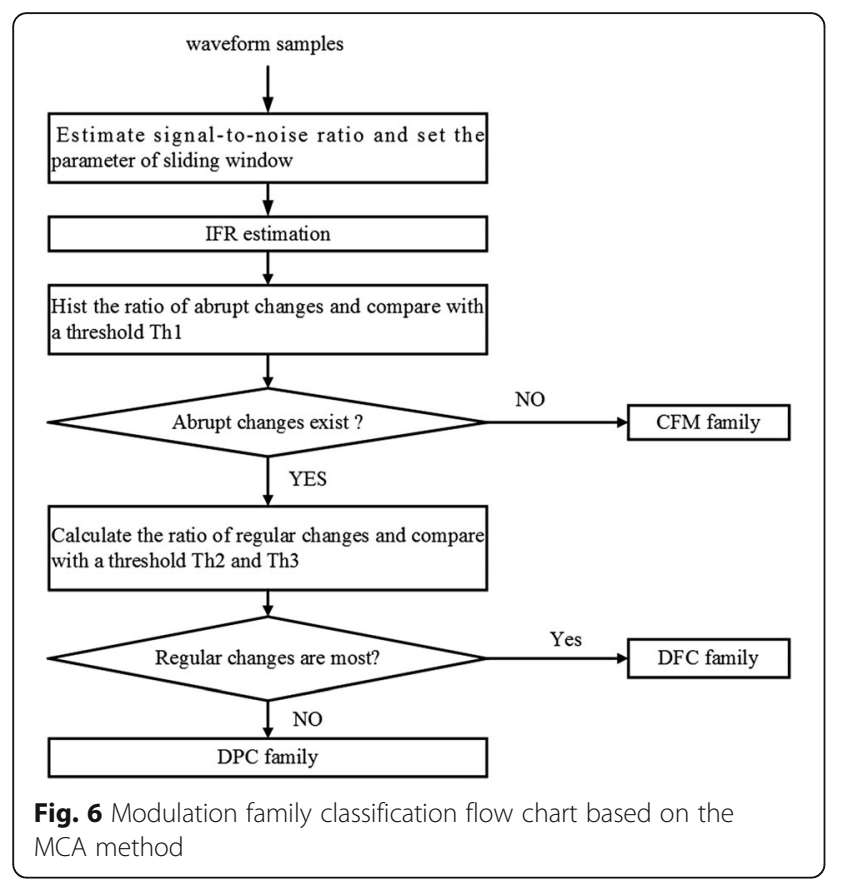

MSEs of the M2M4 algorithm approaches its theoretical variance for $\mathrm{SNR}>-5 \mathrm{~dB}$ and $\mathrm{SNR}<15 \mathrm{~dB}$ [22]. In this paper, we assume that the classification problem is considered when the M2M4 algorithm works well. For the discrete receiving signal in AWGN, it is easy to calculate the 2nd order moment $M_{2}$ and 4th order moment $M_{4}$.

$$
\begin{aligned}
& M_{2}=E\left[r_{n} r_{n}^{*}\right] \cong \frac{1}{N} \sum_{n=0}^{N-1}\left|r_{n}\right|^{2} \\
& M_{4}=E\left[\left(r_{n} r_{n}^{*}\right)^{2}\right] \cong \frac{1}{N} \sum_{n=0}^{N-1}\left|r_{n}\right|^{4}
\end{aligned}
$$

where $E[\bullet]$ is the mathematical expectation of the

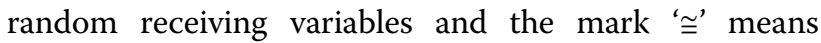
"approximately equals to". Thus the estimated SNR is expressed as

$$
S \hat{N} R=\frac{\sqrt{\left|2 M_{2}^{2}-M_{4}\right|}}{M_{2}-\sqrt{\left|2 M_{2}^{2}-M_{4}\right|}}
$$

Given a requested MSE $\sigma_{I F R}^{2}$ of the IFR estimation, the length of the window can be obtained by (19).

$$
\widehat{N}_{w}=\left\lceil\sqrt[5]{\frac{1}{\pi^{2}} \frac{90 f_{s}^{4}}{\sigma_{I F R}^{2} S \hat{N} R}}\right\rceil
$$

where the mark ' $\ulcorner\cdot\urcorner$ ' is the top integral function which gives the minimum integral value that satisfies the equation. From the above equation, we can calculate that if the estimated $S \hat{N} R$ has a maximum error of $0.5 \mathrm{~dB}$, then the length of the sliding window has a maximum error of less than $3 \%$. Therefore, the length of the sliding window can be estimated accurately when the M2M4 algorithm works well.

\subsubsection{IFR estimation using a sliding window}

To realize the IFR estimation by (17), some parameters should be considered according to the signal processing capability and they are adjustable for the algorithm designer. These parameters are:

- The maximum value $I F R_{\max }$ of the possible IFRs,

- The minimum value $I F R_{\min }$ of the possible IFRs,

- The IFR increment or the step length $\triangle I F R$, and

- The length sliding window $N_{\text {win }}$, an odd number that satisfies $N_{\text {win }} \geq \widehat{N}_{w}$.

For a given time instant $n_{c}$, the IFR can be calculated using the window with length $\widehat{N}_{w}$.

$$
\operatorname{IFR}\left(n_{c}\right)=\frac{f_{s}^{2}}{2 \pi} \underset{\omega}{\operatorname{argmax}}\left|\operatorname{IFRT}(\omega) * \mathbf{y}_{w i n}\left(n_{c}\right)\right|
$$

where $\mathbf{y}_{\text {win }}\left(n_{c}\right) \in \mathbf{C} \frac{\left(N_{\text {win }}+1\right)}{2} \times 1$, the $m$ th element is

$$
\left[\mathbf{y}_{\text {win }}\left(n_{c}\right)\right]_{m}=r\left(n_{c}-m\right) r\left(n_{c}+m\right), \quad 0 \leq m \leq \frac{N_{\text {win }}+1}{2}
$$

and IFRT $\in \mathbf{C}^{N_{I F R} \times \frac{\left(N_{\text {win }}+1\right)}{2}}$ is a transform matrix to calculate the IFR and $N_{I F R}$ is the number of possible IFR elements.

$$
[\operatorname{IFRT}(\omega)]_{m n}=\exp \left(-j \omega_{m}(n-1)^{2}\right)
$$

where $\omega_{m}=2 \pi I F R_{m} / f_{s}^{2}$. As long as the IFR values caused by the abrupt changes range from $I F R_{\min }$ to $I F R_{\max }$, the proposed MCA method will perform well.

\subsubsection{Abrupt changes analysis and the thresholds in IMOP family recognition}

We set the thresholds in the MCA method through Monte Carlo simulations for a broad span of SNR and modulations. The threshold Th1 follows two strategies and is a trade-off between two sub-thresholds, $T h_{01}$ and $T h_{02} . T h_{01}$ is an SNR-dependent threshold and $T h_{02}$ is an SNR-independent threshold related to the signal processing capability. For a signal in the CFM family, there are no abrupt changes in the IFR curve in theory. However, some outliers that seem like abrupt changes may appear when the signal is disturbed by noise. Assuming that the ratio of abrupt changes in the IFR curve is $\alpha$ and Th1 is used to distinguish it from the other two IMOP families. According to the properties of a CFM 
modulation, the threshold should be a slowly decreasing function of SNR values and always positive. By the Monte Carlo simulations for a broad span of SNR values, a possible threshold can be defined as

$$
T h_{01}=\rho \exp (-S N R) / S N R
$$

where $\rho \in(0,1)$ is a small coefficient. On the other hand, the SNR-independent threshold $T h_{02}$ is related to the maximum ratio $\eta_{1}$ of abrupt changes in the IFR curve for a CFM modulation at low SNR.

$$
T h_{02}=\eta_{1}
$$

Thus, the threshold Th1 should be

$$
T h 1=\min \left(T h_{01}, T h_{02}\right)
$$

where $\min (\bullet)$ is the minimum value function. If $\alpha>T h 1$, it means that the abrupt changes caused by modulations are detected.

The thresholds Th2 and Th3 are used to determine whether an IMOP belongs to DFC family or DPC family. The ratio of the regular changes in all abrupt changes $\beta$ and the number of change segments $N_{\text {seg }}$ are defined here. From the analyses above, we conclude that $\beta$ is close to 1 when the IMOP belongs to the DFC family at high SNR while it approximates 0 when the IMOP is a DPC modulation. Th2 is a threshold to evaluate the proportion of regular changes in all abrupt changes.

$$
T h 2=\eta_{2}
$$

According to the majority rule, there is $\eta_{2} \in(0.5,1)$ for an IMOP in the DFC family. In order to increase the reliability of recognition, the threshold Th3 is set to compare with the number of change segments $N_{\text {seg. }}$. While the window slides along the received signal samples, there is at most one change segment caused by the adjacent sub-pulses for signal in the DFC family. It means that the maximum number of change segments for DFC modulation will not be bigger than the number of subpluses. However, as the signal processing is noncooperative, the number of sub-pulses is difficult to obtain. Here, we assume that the number of points of the sliding window is less than that of a sub-pulse; therefore, we have

$$
T h 3=N / N_{\text {win }} .
$$

Thus, a modulation type belongs to the DFC family will be recognized if $\beta>$ Th 2 and $N_{\text {seg }}<$ Th3.

The thresholds $T h_{01}$ and Th3 change their values autonomously for different intercepted signals with different SNRs while $T h_{02}$ and $T h 2$ are optional empirical thresholds which can improve classification performance according to noise level. So far, the IMOP families are recognized using the MCA method.

\subsection{Accurate modulation classification within modulation families}

The MCA method based on the abrupt changes can only classify an IMOP into a modulation family. If more information of an intercepted pulse is wanted, it is necessary to carry out an accurate modulation classification within a certain modulation family. Thus, it becomes a new classification problem. Four basic IMOPs including the CF, LFM, BPC, and BFC modulations are considered here as examples. The modulation features are extracted from the instantaneous phase and instantaneous frequency law (IFL) curve which is calculated according to (14) and (17).

$$
\widehat{I F L}\left(t_{i}\right)=\underset{f}{\operatorname{argmax}}\left|\int_{\mathbb{R}} G C M_{2}^{1}\left[s, t_{i}\right](\tau) e^{-j 2 \pi f \tau} d \tau\right|, \quad \tau \in\left[-T_{w}, T_{w}\right]
$$

For the BFC modulated signal in the DFC family, we can recognize it by counting the number of frequencies in the IFL curve. For the BPC modulated signal in the $\mathrm{BPC}$ family, we can recognize it by determining whether the squared signal shows properties of a CF modulation. For an IMOP in the CFM family, we use the piecewise linear fitting method to the IFL curve. The mean square errors are derived between the instantaneous frequency and its least squares linear fitting. The corresponding classification flow is carried out as follows.

(1) Estimate the IFL curves and do linear fitting to get the corresponding initial frequency $f_{L F O}$ and chirp rate $k_{L F 0}$.

(2)Divide the IFL into two equal pieces and do linear fitting to each piece to get $f_{L F 1}, k_{L F 1}$ and $f_{L F 2}, k_{L F 2}$, and here, we suppose that $k_{L F 1} \geq k_{L F 2}$. $k_{\max }$ is the maximum of the two absolute values $\left|k_{L F 1}\right|$ and $\left|k_{L F 2}\right|$.

(3) Calculate the ratios, $r_{f 1}=f_{L F 1} / f_{L F 0}, r_{f 2}=f_{L F 2} / f_{L F 0}$, $r_{k}=k_{L F 1} / k_{L F 2}$

Table 1 Parameters for radar pulse generation and signal processing

\begin{tabular}{ll}
\hline Parameters & Values \\
\hline Maximum processing points & 1000 \\
Sampling frequency & $100 \mathrm{MHz}$ \\
Carrier frequency & $6 \mathrm{MHz}$ \\
Code rate for BPC and QPC & $4 \mathrm{MHz}$ \\
Code rate for PPC & $5 \mathrm{MHz}$ \\
Code rate for DFC & $1 \mathrm{MHz}$ \\
Chirp rate for LFM & $10 \mathrm{MHz} / \mu \mathrm{s}$ \\
Number of phase increments for PPC & 64 \\
SNR & {$[-7,10] \mathrm{dB}$} \\
Number of Monte Carlo experiments & 500 \\
Possible IFR range & {$[-15,15] \mathrm{MHz} / \mu \mathrm{s}$} \\
Given RMSE of IFR estimation & $0.5 \mathrm{MHz} / \mu \mathrm{s}$ \\
\hline
\end{tabular}


Table 2 The IMOPs for the MCA method in the simulation

\begin{tabular}{ll}
\hline Modulation family & IMOPs \\
\hline CFM & CF, LFM, VFM, SFM \\
DFC & BFC, Costas coding, LFM-BFC, \\
DPC & BPC, QPC, Frank, P1, P2, P3, P4, LFM-BPC, BPC-BFC \\
\hline
\end{tabular}

(4) If $r_{t h 1} \leq r_{f 1} \leq r_{t h 2}, r_{t h 1} \leq r_{f 2} \leq r_{t h 2}$, and $k_{\max } \leq k_{\max T h}$, the IMOP is determined as a CF modulation.

Otherwise, if $r_{t h 1} \leq r_{k} \leq r_{t h 2}$, the IMOP is determined as an LFM.

The thresholds $r_{t h 1}, r_{t h 2}$ and $k_{\max T h}$ are determined according to the maximum errors which can be designed at the initial stage of the signal processing procedure. The accurate modulation classification is realized based on the known modulation families.

\section{Results and discussion}

Two experiments are designed to demonstrate the effectiveness of the proposed method. The first one is used to check the performance of the MCA method and radar pulses are classified into different IMOP families. The second one is designed to classify four commonly used IMOPs by accurate classification within their IMOP families and the results are compared with the AF-based method [15]. In these two experiments, we evaluate the performances by the probability of successful modulation family recognition (PSMFR) and the probability of successful modulation recognition (PSMR) which are both calculated using the Monte Carlo experiments. The simulation parameters are shown in Table 1.

In the MCA method, the coefficient $\rho$ in $T h_{01}$ is set as 0.25 and the maximum ratio of abrupt changes $\eta_{1}$ for the CFM components in $T h_{02}$ is set as 0.2 , and the proportion of regular changes in all abrupt changes for the DFC components $\eta_{2}$ is set as 0.7 . In the accurate modulation classification part, threshold $r_{t h 1}$ is set as 0.8 while $r_{\text {th2 }}$ is set as 1.2. The maximum value $k_{\max T h}$ from a CF modulated signal is set as $10^{11} \mathrm{~Hz} / \mathrm{s}$ which is related to minimum chirp rate for an LFM signal. These thresholds are verified by a broad band of experiments.

\subsection{Autonomous classification for modulation families}

The first experiment gives the performance of the proposed MCA method. The simulation adopts modulations in Table 2. The three kinds of hybrid modulations are the LFM-BFC modulation [10] in the DFC family and the LFM-BPC $[8,9]$ and the BPC-BFC [11] modulations in the DPC family.

Figure 7 gives the probabilities of successful modulation family recognition (PSMFRs) for the simulated
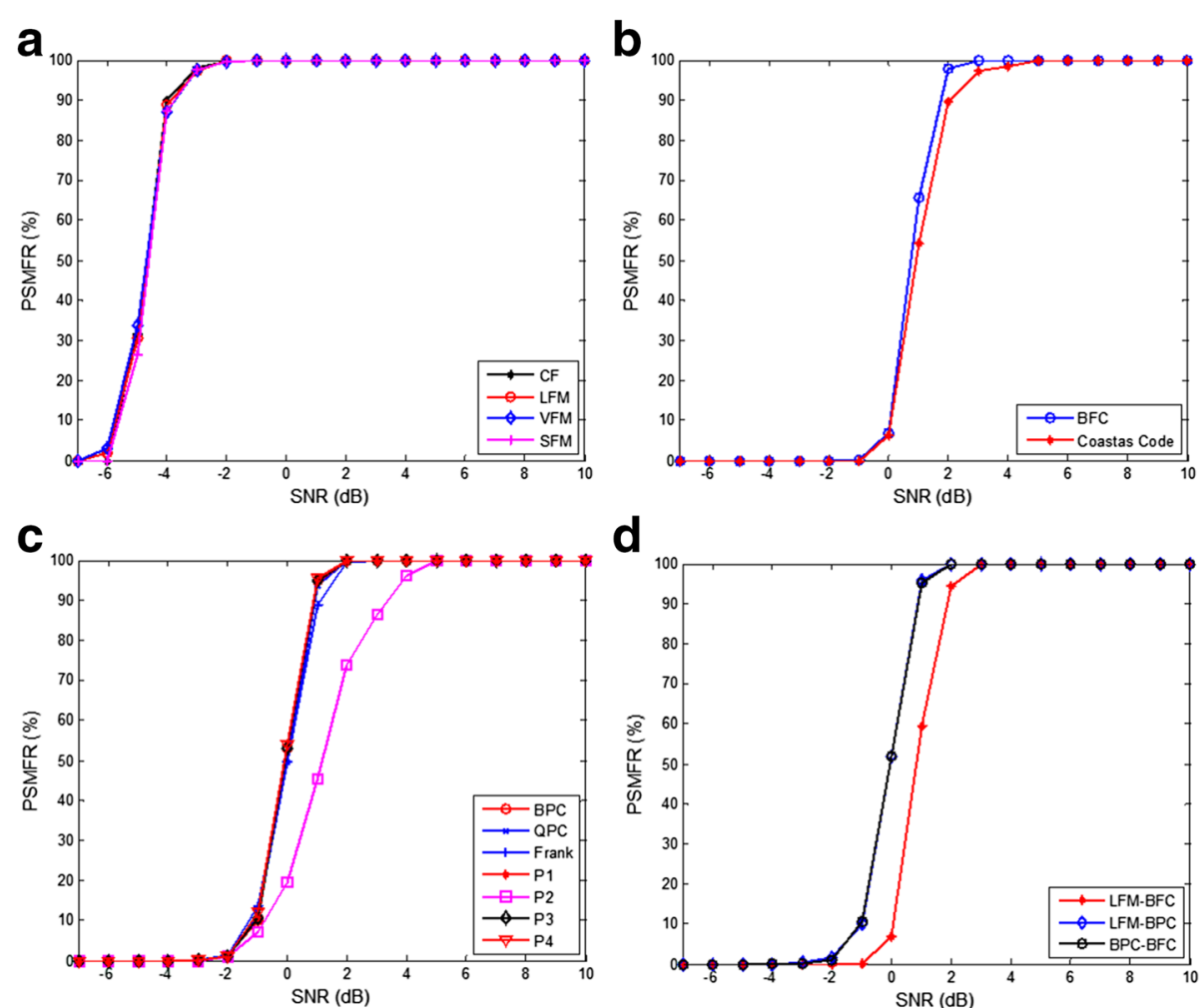

Fig. 7 PSMFRs of radar pulse modulations at different SNR values: (a) Basic IMOPs in CFM family; (b) Basic IMOPs in DFC family; (c) Basic IMOPs in DPC family; and (d) Hybrid IMOPs in the DFC and DPC families 
IMOPs. Figure 7a shows that the PSMFRs for the signals in the CFM family reach $98 \%$ when the SNR is above $-3 \mathrm{~dB}$. These signals have similar PSMFRs and the reason is that they are classified by the same threshold Th1. Figure 7b shows the PSMFRs of BFC and Costas coded signals in the DFC family, and they can be classified well when the SNR is above $3 \mathrm{~dB}$. In Fig. 7c, several IMOPs in the DPC family are classified and can be classified well when the SNR is above $4 \mathrm{~dB}$. Figure $7 \mathrm{~d}$ gives the PSMFRs of three hybrid modulations, and the proposed method achieves a successful probability of $95 \%$ for the three kinds of signals when the SNR is above $2 \mathrm{~dB}$. This experiment shows that the MCA method can give partial information about the modulation family for various kinds of IMOPs.

\subsection{Accurate classifications for four basic radar pulse modulations}

This experiment is designed to verify how the proposed method performs compared with the method based on the AF properties [15]. As the comparison method can only deal with a limited number of IMOPs, we do our experiment here for the four commonly used basic IMOPs, the CF, LFM, BPC, and BFC modulations. For a better comparison, we choose the pulse width as $5 \mu \mathrm{s}$ which has been used in [15]. The PSMRs are calculated using 1000 Monte Carlo trials for each SNR value. The PSMRs for the above four kinds of IMOPs are shown in Fig. 8.

For the CF signals, Fig. 8a shows that the proposed method and the comparison method have nearly the same PSMRs which are greater than $96 \%$ when the SNR is above $-2 \mathrm{~dB}$. The PSMRs for the LFM signals approaches $100 \%$ for both of the two signals when the SNR is above $0 \mathrm{~dB}$ in Fig. $8 \mathrm{~b}$. However, the proposed method performs better than the comparison method when the SNR decreases. In Fig. 8c, we see that the PSMRs are greater than $97 \%$ when the SNR is above $2 \mathrm{~dB}$. The comparison method has slow increasing PSMRs when the SNR values ranges from -6 to $2 \mathrm{~dB}$ while the proposed method has steep rising PSMRs when the SNR is close to $1 \mathrm{~dB}$. For the BPC signals, the PSMRs for both methods are greater than $98 \%$ as Fig. $8 \mathrm{~d}$ shows when the SNR is above $2 \mathrm{~dB}$ and the proposed method has a slightly better performance than the comparison method when the SNR is smaller than $2 \mathrm{~dB}$. Figure 8 shows that the proposed method and the comparison method differ slightly only when the SNR is below $2 \mathrm{~dB}$.

From the two experiments above, we know that if an IMOP has features matched with a known database, it
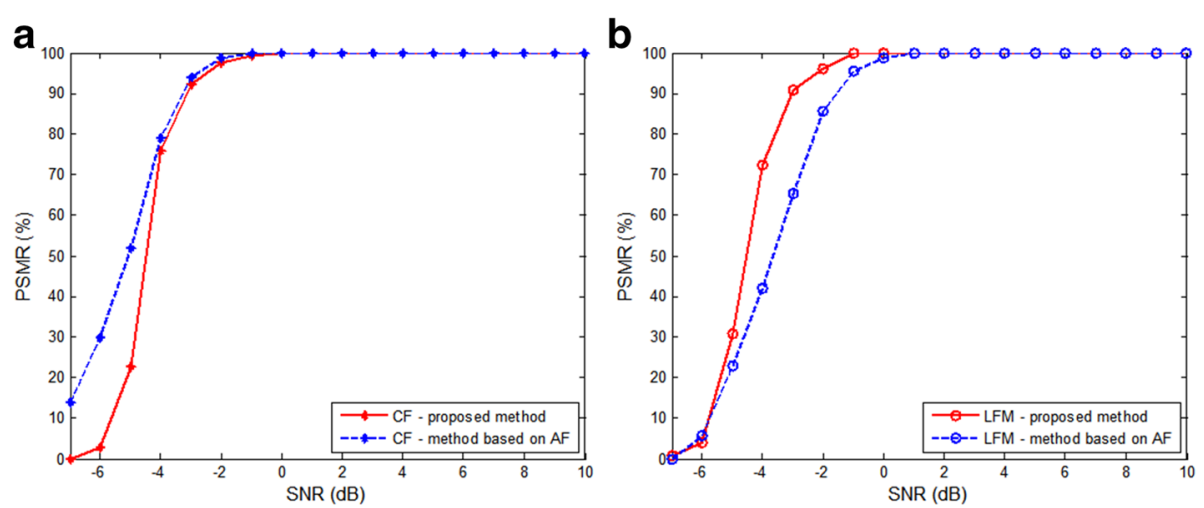

C

d
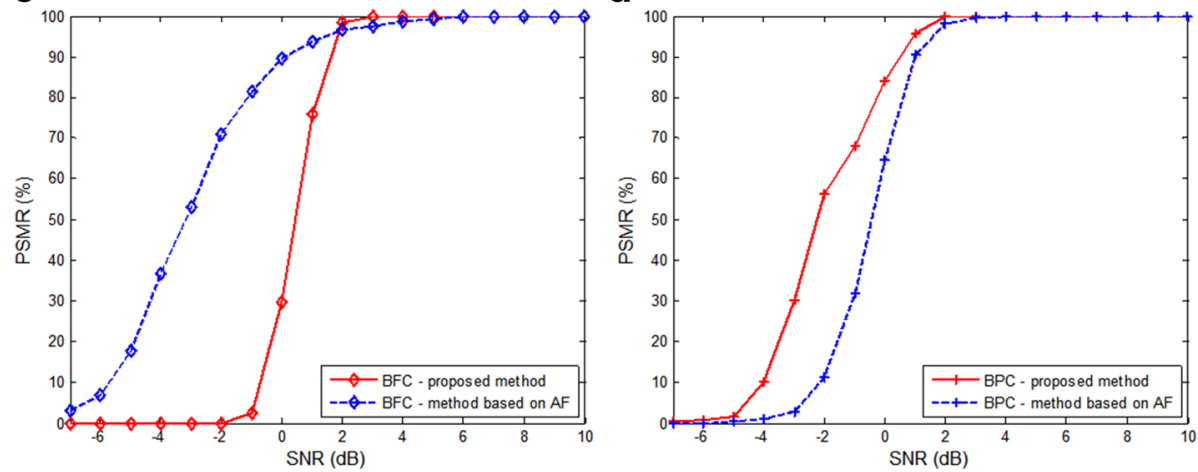

Fig. 8 PSMRs of four basic radar pulse modulations at different SNR values: (a) CF; (b) LFM; (c) BFC; and (d) BPC 
can be recognized with a similar overall performance to the method based on the AF properties whose PSMR is more than $96 \%$ when the SNR is above $2 \mathrm{~dB}$. However, if an IMOP does not belong to a known database, the proposed method can still work and obtain partial information by classifying it into a modulation family while the conventional methods fail to work. In general, the proposed method gives a different classification structure for classification methods of radar pulse modulations. What is more, the accurate modulation classification part can be extended easily within a certain modulation family.

\section{Conclusions}

A two-stage pulse modulation classification method which contains a "modulation family classification" and an "accurate classification" is presented. The former stage is autonomously realized based on the proposed MCA classification method and different IMOPs are classified into three modulation families based on the abrupt change features extracted from the IFR curves. A final decision is done by the latter stage which provides supplementary information for accurate classifications. This method can deal with a broad range of IMOPs and gives partial or complete modulation information while the existing methods give complete information for only a limited number of IMOPs. The theoretical analysis is validated by two designed experiments. The proposed method gives a different classification structure for radar pulse modulations and can be extended easily. Future work will focus on the study of different realization methods for the MCA structure and the extension of the accurate modulation recognition part to some complicated or hybrid modulations.

\section{Abbreviations \\ AF: Ambiguity function; AMC: Automatic modulation classification; AMFRR: Autonomous modulation family recognition rate; AWGN: Additive white Gaussian noise; BFC: Binary frequency codes; BPC: Binary phase codes; CFM: Continuous frequency modulation; CRB: Cramer-Rao bound; DFC: Discrete frequency coded; DPC: Discrete phase coded; EW: Electronic warfare; FSK: Frequency shift keying; GCM: Generalized complex moment; GRPD: General representation of phase derivative; IFL: Instantaneous frequency law; IFR: Instantaneous frequency rate; IMOP: Intentional modulation on pulse; LFM: Linear frequency modulation; LPI: Low probability of intercept; MCA: Modulation components analysis; MIMO: Multiple-input multiple-output; M2M4: 2nd and 4th order moments; NLFM: Nonlinear frequency modulations; PSK: Phase-shift keying; PPC: Polyphase codes; PSMFR: Probability of successful modulation family recognition; PSMR: Probability of successful modulation recognition; QPC: Quadriphase codes; SFM: Sine frequency modulation; SNR: Signal-to-noise ratio; UMOP: Unintentional modulation on pulse; VFM: V-shape frequency modulation}

\section{Acknowledgements}

The authors thank the National Natural Science Foundation of China for their support for the research work. The authors also thank the reviewers for their suggestions and corrections to the original manuscript.

\section{Funding}

The work is supported by the National Natural Science Foundation of China, Grant No. 61571088 and No. 61172116.

\section{Authors' contributions}

The work presented here was carried out in collaboration between all authors. PW proposed the idea and structure of the autonomous classification and wrote the whole paper. ZQ and JZ found out the related literatures and co-designed the experiments. Professor BT gave important suggestions for the structure and experiment results of the paper. All authors have contributed to, seen, and approved the manuscript.

\section{Competing interests}

The authors declare that they have no competing interests.

Received: 29 April 2016 Accepted: 2 September 2016

Published online: 13 September 2016

\section{References}

1. RG Wiley, ELINT, The Interception and Analysis of Radar Signals (Artech House, Norwood, 2006), pp. 1-8

2. DL Adamy, EW 102: A Second Course in Electronic Warfare (Horizon House, Norwood, 2004), pp. 48-54

3. PE Pace, Detecting and Classifying Low Probability of Intercept Radar, 2nd edn. (Artech House, Norwood, 2009), pp. 1-37

4. S Haykin, Cognitive radar: a way of the future. IEEE Signal Process. Mag. 23(1), 30-40 (2006)

5. SP Sira, Y Li, AP Suppappola, D Morrell, D Cochran, M Rangaswamy, Waveform-agile sensing for tracking. IEEE Signal Process. Mag. 26(1), 53-64 (2009)

6. W Huleihel, J Tabrikian, R Shavit, Optimal adaptive waveform design for cognitive MIMO radar. IEEE Trans. Signal Process. 61(20), 5075-5089 (2013)

7. S Wang, Q He, Z He, LFM-based waveform design for cognitive MIMO radar with constrained bandwidth. EURASIP J. Adv. Signal Process. 2014, 89 (2014)

8. DJ Lynch, Introduction to RF Stealth (SciTech Publishing, Releigh, 2004), pp. 336-343

9. M Ngwar, J Wight, Phase Coded Linear Frequency Modulated Waveform for Low Cost Marine Radar System, in Radar Conference (IEEE, Washington DC, 2010), pp. 1044-1049

10. M Kronauge, $\mathrm{H}$ Rohling, New chirp sequence radar waveform. IEEE Trans. Aero. Electron. Syst. 50(4), 2870-2877 (2014)

11. JA Lemieux, FM Ingels, Analysis of FSKJPSK Modulated Radar Signals Using Costas Arrays and Complementary Welti Code.In Radar Conference (IEEE, Arlington VA, 1990), pp. 589-594

12. OA Dobre, A Abdi, YB Ness, W Su, Survey of automatic modulation classification techniques: classical approaches and new trends. IET Commun. 1(2), 137-156 (2007)

13. GL Risueño, J Grajal, OY Ojeda, Atomic decomposition-based radar complex signal interception. IEE Proc. Radar Son. Nav. 150(4), 323-331 (2003)

14. H Lunden, V Koivunen, Automatic radar waveform recognition. IEEE J. Sel. Top. Signal Process. 1(1), 124-136 (2007)

15. D Zeng, H Xiong, J Wang, B Tang, An approach to intra-pulse modulation recognition based on the ambiguity function. Circ. Syst. Signal Process. 29(6), 1103-1122 (2010)

16. D Zeng, X Zeng, G Lu, B Tang, Automatic modulation classification of radar signals using the generalized time-frequency representation of Zhao, Altas and Marks. IET Radar Son. Nav. 5(4), 507-516 (2011)

17. D Zeng, X Zeng, H Cheng, B Tang, Automatic modulation classification of radar signals using the Rihaczek distribution and Hough transform. IET Radar Son. Nav. 6(5), 322-331 (2012)

18. PO Shea, A fast algorithm for estimating the parameters of a quadratic FM signal. IEEE Trans. Signal Process. 52(2)، 385-393 (2004)

19. C Cornu, S Stankovic, C loana, A Quinquis, L Stankovic, Generalized representation of phase derivatives for regular signals. IEEE Trans. Signal Process. 55(10), 4831-4838 (2007) 
20. F Digne, C Cornu, A Baussard, A Khenchaf, D Jahan, in IET International Conference on Radar Systems (Radar 2012). Use of short-term polynomial phase estimation for new electronic warfare systems, (IEEE, Glasgow, UK, 2012), pp. 1-6

21. DR Pauluzzi, NC Beaulien, A comparison of SNR estimation techniques for the AWGN channel. IEEE Trans. Commun. 48(10), 1681-1691 (2000)

22. LV Roberto, M Carlos, Sixth-order statistics-based non-data-aided SNR estimation. IEEE Commun. Lett. 11(4), 351-353 (2007)

Submit your manuscript to a SpringerOpen ${ }^{\mathcal{D}}$ journal and benefit from:

- Convenient online submission

- Rigorous peer review

- Immediate publication on acceptance

- Open access: articles freely available online

- High visibility within the field

- Retaining the copyright to your article

Submit your next manuscript at $>$ springeropen.com 